\title{
ON CONVEX HULLS AND PSEUDOCONVEX DOMAINS GENERATED BY $q$-PLURISUBHARMONIC FUNCTIONS, PART III
}

\author{
T. PAWLASCHYK AND E. S. ZERON
}

\begin{abstract}
We characterise in this work the $q$-plurisubharmonic functions in terms of the theory of viscosity solutions. We show that an upper semi-continuous function is $q$-plurisubharmonic if and only if its complex Hessian has at most $q$ strictly negative eigenvalues in the viscosity sense. This characterisation is then used to prove that the sup-convolution of a (strictly) $q$-plurisubharmonic function is again (strictly) $q$-plurisubharmonic on a maybe different set of definition. Finally, we use the supremum convolution to deduce a new characterisation for the $q$-pseudoconvex subsets in $\mathbb{C}^{n}$.
\end{abstract}

\section{INTRODUCTION}

This article is a sequel and conclusion to our research previously developed in the works [13] and [14]. Our main objective now is to characterise the $q$-plurisubharmonic functions in terms of the theory of viscosity solutions; and this result is posed and proven in Definition 2.6. Proposition 2.7. and Theorem 2.10 in the following chapter. There we show that an upper semi-continuous function $u$ is $q$ plurisubharmonic if and only if either $u \equiv-\infty$ or its complex Hessian $H^{\mathbb{C}} u$ has at most $q$ strictly negative eigenvalues in the viscosity sense; i.e., if for every $\mathcal{C}^{2}$ smooth test function $\varphi$, the complex Hessian $H^{\mathbb{C}} \varphi$ has at most $q$ strictly negative eigenvalues at those points where $u-\varphi$ attains its maximum. This result was inspired by Alvarez, Lasry, and Lions paper 2, where the authors showed that an upper semi-continuous function $u$ is convex if and only if its real Hessian $H^{\mathbb{R}} u$ has no strictly negative eigenvalues in the viscosity sense. We strongly recommend the manuscripts of Crandall [4, Crandall et al. [5], Koike [10, or Katzourakis [9] for a general presentation on the theory of viscosity solutions.

Date: October 25, 2018.

2010 Mathematics Subject Classification. Primary 32U05, Secondary 35D40, 31C10.

Key words and phrases. (strictly) q-plurisubharmonic functions, viscosity subsolutions, Moreau sup-convolution.

Research supported by the Deutscher Akademischer Austauschdienst (DAAD) and Conacyt México under the PPP Proalmex Project No. 51240052. The first author was supported by the Deutsche Forschungsgemeinschaft (DFG) under the grant SH 456/1-1, Pluripotential Theory, Hulls and Foliations; and the second author was supported by Cinvestav del IPN in México while he was in a sabbatical leave in CRM de l'Université de Montréal in Canada.

The original manuscript was submited for its publication to the Journal of Mathematical Analysis and Applications. 
On the other hand, in 1984 Słodkowski introduced a product based supremum convolution for $q$-plurisubharmonic functions $u$ in order to build non-increasing sequences of continuous $q$-plurisubharmonic functions $\left\{u_{k}\right\}_{k=1}^{\infty}$ that converge pointwise to the original $u$, when $k \rightarrow \infty$, and such that each $u_{k}$ has almost everywhere second-order derivatives in the Peano sense; see [15].

In the same form, one can apply the sup-convolution introduced by Moreau in 1963 [1], 12 in order to produce continuous approximations to $q$-plurisubharmonic functions, because the $q$-plurisubharmonicity can be characterised in terms of viscosity subsolutions of the elliptic degenerate operator $\ominus$ given in Lemma 2.4 and one has the so called magic property: the sup-convolution of a viscosity subsolution is again a viscosity subsolution. Besides, it is well known that the sup-convolution takes upper semi-continuous functions $u$ (which may not be integrable) and in general produces continuous ones $u^{\Phi}$. For example, if $\Phi$ is of the form $-k\|\cdot\|^{2}$, the sup-convolution then satisfies $u^{\Phi} \geq u$, the sum $u^{\Phi}+k\|\cdot\|^{2}$ is convex, and $\left\{u^{\Phi}\right\}_{k=1}^{\infty}$ is a non-increasing sequence which converges pointwise to $u$ as $k \rightarrow \infty$; see Chapter 4 of [9] or Section 11 of 4. We include a fast introduction to the sup-convolution and its properties in the third section of this work for the sake of completeness, but the reader who already knows the results can skip this section.

We conclude this work by showing in Proposition 4.1 and the subsequent corollaries a variation of the magic property: given any $q$-plurisubharmonic function $u$ and a quadratic real-valued polynomial $g(y)=y^{t} \mathcal{A} \bar{y}$ for a fixed Hermitian matrix $\mathcal{A}$, the sup-convolution $[u+g]^{\Phi}-g$ is again $q$-plurisubharmonic on a maybe different set of definition. As an application we deduce that the sup-convolution of a strictly $q$-plurisubharmonic function is again strictly $q$-plurisubharmonic; moreover, we also deduce a new characterisation for the $q$-pseudoconvex subsets in $\mathbb{C}^{n}$. This latter result extends a list of characterisations and properties of $q$-pseudoconvex sets we collected in [13] and [14].

\section{2. $q$-PLURISUBHARMONIC FUNCTIONS AS VISCOSITY SUBSOLUTIONS}

We characterise in this section the $q$-plurisubharmonic functions $u$ as viscosity subsolutions to the elliptic degenerate operator $u \mapsto \ominus\left(H^{\mathbb{C}} u\right)$ introduced in the Lemma 2.4 below; i.e., $u$ is $q$-plurisubharmonic if and only if either $u \equiv-\infty$ or its complex Hessian $H^{\mathbb{C}} u$ has at most $q$ strictly negative eigenvalues in the viscosity sense. The main objective is to apply the results of the viscosity solutions theory originally developed by Crandall, Lions, and collaborators. We recommend in particular the manuscripts of Crandall [4, Crandall et al. [5], Koike [10, and Katzourakis 9 .

From now on $\langle x, y\rangle=\sum_{k=1}^{n} \operatorname{Re}\left(x_{k} \overline{y_{k}}\right)$ denotes the standard real inner product between vectors $x$ and $y$ in $\mathbb{C}^{n}$, so that $\sqrt{\langle x, x\rangle}$ is the Euclidean norm $\|x\|$. In the same way, the notation $u \equiv-\infty$ means that $u$ is the function identically equal to $-\infty$ on its set of definition; the set $B_{\rho}(x) \subset \mathbb{C}^{n}$ is the standard Euclidean open ball of radius $\rho>0$ and with centre on $x$; while $U \subset \mathbb{C}^{n}$ always stands for a non-empty open set.

We need to recall the definition of a $q$-plurisubharmonic function introduced by Hunt and Murray; see for example [8]. In particular, inspired by Słodkowski's Lemma 2.2 below, we prefer to introduce first the notion of subpluriharmonic functions instead of the $(n-1)$-plurisubharmonic ones. 
Definition 2.1. Let $u: U \rightarrow[-\infty, \infty)$ be an upper semi-continuous function defined on some open set $U \subset \mathbb{C}^{n}$.

(1) The function $u$ is subpluriharmonic on $U$ if and only if either $u \equiv-\infty$ on $U$ or $u+h$ has the maximum property for every continuous plurisubharmonic function $h$ defined in a neighborhood of any compact ball $\bar{B} \subset U$; i.e., the sum $u+h$ attains its maximum at the boundary $b B$.

(2) For any integer $q$ with $0 \leq q<n$, the function $u$ is $q$-plurisubharmonic on $U$ if and only if it is subpluriharmonic on the intersection $U \cap \pi$ with every possible $(q+1)$-dimensional complex affine space $\pi$ in $\mathbb{C}^{n}$. Subpluriharmonic functions coincide with the $(n-1)$-plurisubharmonic ones.

(3) Finally, we say by convention that any upper semi-continuous function is $N$-plurisubharmonic whenever $N \geq n$.

(4) The set of all $q$-plurisubharmonic functions on $U$ is denoted by $\operatorname{PSH}_{q}(U)$.

Słodkowski showed in Lemma 4.4 of [16] that conditions in the point (11) above can be relaxed; namely, it is sufficient to consider pluriharmonic polynomials (globally defined on $\mathbb{C}^{n}$ ) instead of plurisubharmonic functions. Recall that a pluriharmonic polynomial (or function) is the real part $\pm \operatorname{Re}(h)$ of a holomorphic one $h$. We include Słodkowski's lemma for the sake of completeness.

Lemma 2.2. [Słodkowski] Let $u: U \rightarrow[-\infty, \infty)$ be an upper semi-continuous function well defined on a non-empty open set $U \subset \mathbb{C}^{n}$. We have that $u$ lies in $P_{S H} H_{n-1}(U)$ if and only if the sum $u-\operatorname{Re}[\wp]$ has the local maximum property for every holomorphic polynomial $\wp: \mathbb{C}^{n} \rightarrow \mathbb{C}$; i.e., given any compact ball $\bar{B}$ in $U$, the new function $u-\operatorname{Re}[\wp]$ attains its maximum in the boundary $b B$.

Proof. See Lemma 4.4 and its proof in pages 122 and 123 of [16].

The characterisation of $q$-plurisubharmonic functions is simple when they are smooth enough, as it was indicated by Hunt, Murray and Słodkowski. We present below a collection of properties of $q$-plurisubharmonic functions, which can be found in various papers and are later used; see for example [8, 15, 7, 6, 13, 14.

Proposition 2.3. Let $q$ and $r$ be a pair of non-negative integers, and $U$ be a non-empty open set in $\mathbb{C}^{n}$.

(1) The 0-plurisubharmonic functions are the classical plurisubharmonic ones. Moreover, $\mathrm{PSH}_{q}(U)$ is contained in $\mathrm{PSH}_{r}(U)$ whenever $q \leq r$.

(2) The q-plurisubharmonicity is a local property; i.e. $u \in P S H_{q}(U)$ if and only if every point $x \in U$ has a neighbourhood $\Omega \subset U$ such that the restriction $\left.u\right|_{\Omega}$ lies in $\operatorname{PSH}_{q}(\Omega)$.

(3) Given $c \geq 0$ in $\mathbb{R}$ and two functions $u \in P S H_{q}(U)$ and $v \in P S H_{r}(U)$,

$$
\begin{array}{ll}
c u \in P S H_{q}(U), & \max \{u, v\} \in P S H_{\max \{q, r\}}(U), \\
u+v \in P S H_{q+r}(U), & \min \{u, v\} \in P S H_{q+r+1}(U) .
\end{array}
$$

(4) If $u_{1} \geq u_{2} \geq \ldots$ is a non-increasing sequence of functions in $\operatorname{PSH}_{q}(U)$, then the point-wise limit $\lim _{k \rightarrow \infty} u_{k}$ lies in $P S H_{q}(U)$.

(5) Given a collection $\left\{u_{j}\right\}_{j \in J}$ in $P_{S H_{q}}(U)$ which is locally bounded from above at each point in $U$, the upper semi-continuous regularisation

$$
z \mapsto\left[\sup _{j \in J} u_{j}\right]^{\star}(z)=\limsup _{\zeta \rightarrow z}\left[\sup _{j \in J} u_{j}(\zeta)\right] \text { lies in } P S H_{q}(U) \text {. }
$$


(6) Assume that both $0 \leq q<n$ and the open set $U \subset \mathbb{C}^{n}$ is bounded. Then, every function $u \in P S H_{q}(U)$ that is upper semi-continuous up to the boundary bU also satisfies the maximum principle; i.e.,

$$
\max _{\bar{U}} u=\max _{b U} u \text {. }
$$

(7) $A \mathcal{C}^{2}(U)$-smooth function $u$ is q-plurisubharmonic on $U$ if and only if its complex Hessian below has at most $q$ strictly negative eigenvalues at each point $z \in U$,

$$
H^{\mathbb{C}} u:=\left[\frac{\partial^{2} u}{\partial z_{k} \partial \overline{z_{\ell}}}\right]_{k, \ell=1, \ldots, n} .
$$

We can now proceed with the main objective of this section; i.e, we characterise the $q$-plurisubharmonic functions as those upper semi-continuous functions whose complex Hessian has at most $q$ strictly negative eigenvalues in the viscosity sense. To do so, we need to show first that the number of strictly negative eigenvalues is an elliptic degenerate operator acting on the set of Hermitian matrices.

Lemma 2.4. Let $\ominus(\mathcal{A})$ be the number of strictly negative eigenvalues of the Hermitian matrix $\mathcal{A} \in \mathbb{C}^{n \times n}$, and $\oplus(\mathcal{A})$ be its number of strictly positive eigenvalues. The operators $\ominus$ and $-\oplus$ are both elliptic degenerate; i.e., the inequalities below hold for all Hermitian matrices $\mathcal{A} \geq \mathcal{B}$,

$$
\ominus(\mathcal{A}) \leq \ominus(\mathcal{B}) \quad \text { and } \quad \oplus(\mathcal{A}) \geq \oplus(\mathcal{B}) .
$$

Notice that neither the operator $\ominus$ nor $\oplus$ is continuous and that the latter sign " $\geq$ " designates the Löwner partial order in the set of Hermitian matrices; i.e., $\mathcal{A} \geq \mathcal{B}$ whenever $\mathcal{A}-\mathcal{B}$ is positive semidefinite.

Proof. This is a simple consequence of Theorem 7.9 of [19, because for any two Hermitian matrices $\mathcal{A} \geq \mathcal{B}$ with ordered eigenvalues:

$$
\lambda_{1}(\mathcal{A}) \geq \ldots \geq \lambda_{n}(\mathcal{A}) \quad \text { and } \quad \lambda_{1}(\mathcal{B}) \geq \ldots \geq \lambda_{n}(\mathcal{B})
$$

the inequalities $\lambda_{k}(\mathcal{A}) \geq \lambda_{k}(\mathcal{B})$ hold for all indices $k=1, \ldots, n$.

As in the classical theory of viscosity solutions, the definition for $q$-plurisubharmonic functions in the viscosity sense is naturally motivated by the following result for $\mathcal{C}^{2}$-differentiable functions.

Lemma 2.5. For any integer $q \geq 0$, a function $u \in \mathcal{C}^{2}(U)$ is q-plurisubharmonic on a non-empty open set $U \subset \mathbb{C}^{n}$ if and only if for every function $\varphi \in \mathcal{C}^{2}(U)$, the operator $\ominus\left(H^{\mathbb{C}} \varphi\right) \leq q$ at those points $\hat{p} \in U$ where $u-\varphi$ attains its maximum.

Proof. Firstly assume that for every $\varphi \in \mathcal{C}^{2}(U)$, the operator $\ominus\left(H^{\mathbb{C}} \varphi\right) \leq q$ at those points $\hat{p} \in U$ where $u-\varphi$ attains its maximum. Since $u \in \mathcal{C}^{2}(U)$, we can fix $\varphi \equiv u$, so that $u-\varphi$ obviously attains its maximum (zero) at every point $z \in U$. Whence, $u \equiv \varphi$ lies in $P S H_{q}(U)$, because $\ominus\left(H^{\mathbb{C}} \varphi\right) \leq q$ at every $z \in U$ and according to (7) in Proposition 2.3

On the other hand, suppose now that $u \in \mathcal{C}^{2}(U)$ is $q$-plurisubharmonic and take $\varphi \in \mathcal{C}^{2}(U)$, such that the difference $g=u-\varphi$ attains its maximum at some point $\hat{p} \in U$. Hence, when they are evaluated at $\hat{p}$, both the gradient $\nabla g=0$ vanishes and the real Hessian $H^{\mathbb{R}} g \leq 0$ is negative semi-definite. We assert that the complex Hessian $H^{\mathbb{C}} \varphi$ in (2.1) has at most $q$ strictly negative eigenvalues at $\hat{p}$. 
Recall that the real Hessian $H^{\mathbb{R}} g \in \mathbb{R}^{2 n \times 2 n}$ is symmetric and Hermitian, so that the condition $H^{\mathbb{R}} g \leq 0$ implies $\bar{v}^{t}\left[H^{\mathbb{R}} g\right] v \leq 0$ for every vector $v \in \mathbb{C}^{2 n}$. We can understand the relation between the real and complex Hessians by expanding the complex coordinates into real ones $z_{k}=x_{k}+i y_{k}$, so that $\mathbb{C}^{n} \cong \mathbb{R}^{2 n}$. The following identities are easily satisfied for $z=\hat{p}$ and all vectors $\xi \in \mathbb{C}^{n}$,

$$
\begin{aligned}
\overline{\xi^{t}}\left[\frac{\partial^{2} g}{\partial z_{k} \partial \overline{z_{\ell}}}\right]_{k, \ell} \xi & =\frac{1}{4} \overline{\left[\xi^{t}, i \xi^{t}\right]}\left[\begin{array}{ll}
{\left[\frac{\partial^{2} g}{\partial x_{k} \partial x_{\ell}}\right]_{k, \ell}} & {\left[\frac{\partial^{2} g}{\partial x_{k} \partial y_{\ell}}\right]_{k, \ell}} \\
{\left[\frac{\partial^{2} g}{\partial y_{k} \partial x_{\ell}}\right]_{k, \ell}} & {\left[\frac{\partial^{2} g}{\partial y_{k} \partial y_{\ell}}\right]_{k, \ell}}
\end{array}\right]\left[\begin{array}{c}
\xi \\
i \xi
\end{array}\right] \\
& =\frac{1}{4} \overline{\left[\xi^{t}, i \xi^{t}\right]}\left[H^{\mathbb{R} g}\right]\left[\begin{array}{c}
\xi \\
i \xi
\end{array}\right] \leq 0 .
\end{aligned}
$$

Thus, both inequalities $H^{\mathbb{C}} g \leq 0$ and $H^{\mathbb{C}} u \leq H^{\mathbb{C}} \varphi$ hold, when the complex Hessians are all evaluated at $\hat{p}$. Notice that $\ominus\left(H^{\mathbb{C}} u\right) \leq q$ on $U$, because $u$ is $q$ plurisubharmonic in $U$ and point (7) in Proposition 2.3. A direct application of Lemma 2.4 yields the desired result: $\ominus\left(H^{\mathbb{C}} \varphi\right) \leq q$ at those points $\hat{p}$ where $u-\varphi$ attains its maximum.

We may now present the main definition of this work, the main idea is to rephrase the previous result, so as to get a proper definition for upper semi-continuous functions instead of $\mathcal{C}^{2}$-differentiable ones.

Definition 2.6. Let $q \geq 0$ be an integer, and $u: U \rightarrow[-\infty, \infty)$ be an upper semi-continuous function defined on some open set $U \subset \mathbb{C}^{n}$. We say that $u$ is $q$ plurisubharmonic in the viscosity sense on $U$ whenever either $u \equiv-\infty$ on $U$ or for every $\varphi \in \mathcal{C}^{2}(U)$, the operator $\ominus\left(H^{\mathbb{C}} \varphi\right) \leq q$ at those points $\hat{p}$ where $u-\varphi$ attains its maximum; i.e., the complex Hessian $H^{\mathbb{C}} \varphi$ in (2.1) has at most $q$ strictly negative eigenvalues at those points $\hat{p}$.

In particular, if $u \not \equiv-\infty$, we must only consider those test functions $\varphi$ for which $u-\varphi$ can indeed attain its maximum at some $\hat{p} \in U$, so that $(u-\varphi)(\hat{p})$ and $u(\hat{p})$ are both (finite) real numbers.

For example, any upper semi-continuous function $u$ is $q$-plurisubharmonic in the viscosity sense if $u \equiv-\infty$ or $q \geq n$, since $H^{\mathbb{C}} \varphi$ in (2.1) is an $[n \times n]$-Hermitian matrix when $\varphi \in \mathcal{C}^{2}(U)$.

We now devote the rest of this section to prove that the q-plurisubharmonicities in the viscosity and classical senses coincide for general upper semi-continuous functions. We begin presenting the simpler implication.

Proposition 2.7. Every function $u \in P S H_{q}(U)$ is q-plurisubharmonic in the viscosity sense on $U$, for any integer $q \geq 0$ and non-empty open set $U \subset \mathbb{C}^{n}$.

Proof. The result is trivial when $q \geq n$ or $u \equiv-\infty$ on $U$, because $u$ is $q$-plurisubharmonic in the viscosity sense in both cases. Thus, we assume from now on that $q<n$ and $u(\hat{x}) \in \mathbb{R}$ is finite for some $\hat{x} \in U$. We prove the result by assuming that $u$ is not $q$-plurisubharmonic in the viscosity sense on $U$. Whence, following Definition 2.6 there is a test function $\varphi \in \mathcal{C}^{2}(U)$ such that $u-\varphi$ attains its maximum at some $\hat{p} \in U$, but the operator $\ominus\left(H^{\mathbb{C}} \varphi\right)>q$ at the point $\hat{p}$. In particular, $u(\hat{p}) \in \mathbb{R}$ is finite and the negative complex Hessian $-H^{\mathbb{C}} \varphi$ has at least $q+1$ strictly positive eigenvalues at $\hat{p}$, so that $-H^{\mathbb{C}} \varphi$ has the same number of positive eigenvalues at every point in some small neighbourhood $\Omega_{1}$ of $\hat{p}$. According 
to point (7) in Proposition 2.3 this means that $-\varphi$ is $(n-q-1)$-plurisubharmonic on $\Omega_{1}$.

Choose $\beta>0$ small enough such that $-\varphi(z)-\beta\|z-\hat{p}\|^{2}$ is $(n-q-1)$-plurisubharmonic on a maybe smaller neighbourhood $\Omega_{2}$ of $\hat{p}$; i.e., its complex Hessian has at least $q+1$ positive eigenvalues on $\Omega_{2}$. The sum $(u-\varphi)(z)-\beta\|z-\hat{p}\|^{2}$ then attains its strict global maximum at $\hat{p} \in \Omega_{2}$ and it is $(n-1)$-plurisubharmonic on $\Omega_{2}$. This result is a consequence of point (3) in Proposition 2.3. after considering that $u$ lies in $\operatorname{PSH}_{q}(U)$, that the evaluation $u(\hat{p}) \in \mathbb{R}$ is finite, and that $u-\varphi$ attains its maximum at $\hat{p}$. The existence of a strict global maximum inside $\Omega_{2}$ contradicts the local maximum principle stated in the point (6) of Proposition 2.3, so that $u$ must be $q$-plurisubharmonic in the viscosity sense on $U$.

We need the following result to prove the opposite implication to the previous proposition. This is a classical result in the theory of viscosity solutions, but we include its proof for the sake of completeness.

Lemma 2.8. Let $\Omega \subset U \subset \mathbb{C}^{n}$ be non-empty open sets, and $u: U \rightarrow[-\infty, \infty)$ be an upper semi-continuous function. Assume there is a function $\varphi \in \mathcal{C}^{2}(\Omega)$ such that the restriction of $u-\varphi$ to $\Omega$ attains its maximum at some point $\hat{p} \in \Omega$ and $u(\hat{p}) \in \mathbb{R}$ is finite. Then there is a second function $\psi \in \mathcal{C}^{2}(U)$ such that $u-\psi$ has a strict global maximum at $\hat{p}$ in $U$ and its evaluation $\psi(\hat{p})$, gradient $\nabla \psi(\hat{p})$, and complex Hessian $H^{\mathbb{C}} \psi(\hat{p})$ coincide with the respective evaluation $\varphi(\hat{p})$, gradient $\nabla \varphi(\hat{p})$, and complex Hessian $H^{\mathbb{C}} \varphi(\hat{p})$ of the original function.

Proof. Define the function $\psi_{0}(z):=\varphi(z)+\|z-\hat{p}\|^{6}$. One can easily see that the restriction of $u-\psi_{0}$ to $\Omega$ has a strict global maximum at $\hat{p} \in \Omega$ and its evaluation $\psi_{0}(\hat{p})$, gradient $\nabla \psi_{0}(\hat{p})$, and complex Hessian $H^{\mathbb{C}} \psi_{0}(\hat{p})$ coincide with the respective evaluation $\varphi(\hat{p})$, gradient $\nabla \varphi(\hat{p})$, and complex Hessian $H^{\mathbb{C}} \varphi(\hat{p})$ of the original function. We only need to extend $\psi_{0}$ as a $\mathcal{C}^{2}$-smooth function onto $U$ to conclude the proof. We do this by steeps.

Also define $V_{k}:=\{x \in U: u(x)<k\}$, so that $U=\bigcup_{k=1}^{\infty} V_{k}$. Since $u$ is upper semi-continuous, we have that $V_{k}$ is an open subset of $\mathbb{C}^{n}$ for each integer $k \geq 1$. Take any compact ball $\bar{B} \subset \Omega$ with centre at $\hat{p}$, so that $\psi_{0}$ is $\mathcal{C}^{2}$-smooth in a neighbourhood of $\bar{B}$ and the restriction of $u-\psi_{0}$ to $\bar{B}$ attains its strict global maximum at $\hat{p} \in B$. Since $u$ is upper semi-continuous on $U$, it is bounded from above on the compact ball $\bar{B}$ by a large enough integer $k \geq 1$, so that $\bar{B} \subset V_{k}$. All the previous properties imply that $\psi_{0}$ can be extended from $B$ onto a neighbourhood of $\overline{V_{k}}$ as a $\mathcal{C}^{2}$-smooth function $\psi_{k}$ such that the restriction of $u-\psi_{k}$ to $\overline{V_{k}}$ also has its strict global maximum at $\hat{p}$.

We can obviously carry on with this process, extending $\psi_{j}$ from $V_{j}$ onto a neighbourhood of $\overline{V_{j+1}}$ as a $\mathcal{C}^{2}$-smooth function $\psi_{j+1}$ such that the restriction of $u-\psi_{j+1}$ to $\overline{V_{j+1}}$ attains its strict global maximum at $\hat{p}$. Since $U=\bigcup_{k=0}^{\infty} V_{k}$, the above extension process yields a function $\psi \in \mathcal{C}^{2}(U)$, such that $u-\psi$ also has its strict global maximum at $\hat{p}$ and the functions $\psi$ and $\psi_{0}$ coincide inside the open ball $B$ centred at $\hat{p}$, as we wanted to prove.

A direct consequence of this lemma is that the $q$-plurisubharmonicity in the viscosity sense is a local property.

Corollary 2.9. Let $q \geq 0$ be an integer, and $u: U \rightarrow[-\infty, \infty)$ be an upper semi-continuous function defined on a non-empty open set $U \subset \mathbb{C}^{n}$. 
(1) If every point $x \in U$ has an open neighbourhood $\Omega \subset U$ such that the restriction $\left.u\right|_{\Omega}$ is q-plurisubharmonic in the viscosity sense on $\Omega$, then $u$ is also q-plurisubharmonic in the viscosity sense on $U$.

(2) Given any non-empty open subset $\Omega \subset U$, if $u$ is q-plurisubharmonic in the viscosity sense on $U$, the restriction $\left.u\right|_{\Omega}$ is then q-plurisubharmonic in the viscosity sense on $\Omega$.

Proof. Statement in point (11) trivially holds when $u \equiv-\infty$ on $U$; otherwise, this point is proved by taking any function $\psi \in \mathcal{C}^{2}(U)$ such that $u-\psi$ attains its maximum at some point $\hat{p} \in U$ with $u(\hat{p}) \in \mathbb{R}$. Let $\Omega$ be an open neighbourhood of $\hat{p}$ in $U$ such that the restriction $\left.u\right|_{\Omega}$ is $q$-plurisubharmonic in the viscosity sense in $\Omega$. Since the restriction of $u-\psi$ to $\Omega$ also attains its maximum at $\hat{p} \in \Omega$, Definition 2.6 yields that the operator $\ominus\left(H^{\mathbb{C}} \psi\right) \leq q$ at $\hat{p}$, and so $u$ is $q$-plurisubharmonic in the viscosity sense in $U$ by the same definition.

Result in point (2) trivially holds when $u \equiv-\infty$ on $\Omega$; otherwise, this point is proved by taking any function $\varphi \in \mathcal{C}^{2}(\Omega)$ such that $\left.u\right|_{\Omega}-\varphi$ attains its maximum at some point $\hat{p} \in \Omega$ with $u(\hat{p}) \in \mathbb{R}$. Lemma 2.8 yields the existence of $\psi \in \mathcal{C}^{2}(U)$ such that $u-\psi$ has a strict global maximum at $\hat{p} \in U$ and the complex Hessians $H^{\mathbb{C}} \psi(\hat{p})$ and $H^{\mathbb{C}} \varphi(\hat{p})$ coincide. Since $u$ is $q$-plurisubharmonic in the viscosity sense on $U$, the Hessians $H^{\mathbb{C}} \varphi=H^{\mathbb{C}} \psi$ have at most $q$ strictly negative eigenvalues at $\hat{p}$, and so $\left.u\right|_{\Omega}$ is also $q$-plurisubharmonic in the viscosity sense on $\Omega$.

We may now prove that the q-plurisubharmonicities in the viscosity and classical senses coincide for general upper semi-continuous functions. This result was inspired by Alvarez, Lasry, and Lions paper [2, where they shown that an upper semi-continuous function $u$ is convex if and only if its real Hessian $H^{\mathbb{R}} u$ has no strictly negative eigenvalues in the viscosity sense.

Theorem 2.10. Let $q \geq 0$ be any integer, and $U \subset \mathbb{C}^{n}$ be a non-empty open set. An upper semi-continuous function $u: U \rightarrow[-\infty, \infty)$ is q-plurisubharmonic on $U$ if and only if it is q-plurisubharmonic in the viscosity sense on $U$.

Proof. The result is trivial when $q \geq n$ or $u \equiv-\infty$ on $U$. Moreover, it is already shown in Proposition 2.7 that every $u \in P S H_{q}(U)$ is $q$-plurisubharmonic in the viscosity sense. We assume from now on that both $u \not \equiv-\infty$ is $q$-plurisubharmonic in the viscosity sense on $U$ and $q<n$. We show that $u \in P S H_{q}(U)$ by considering the cases introduced in the main Definition 2.1. The first case happens when $q$ is equal to $n-1$; and so we introduce the test function

$$
\varphi_{\beta}(z):=\operatorname{Re}[\wp](z)-\beta\|z\|^{2} \quad \text { for } \quad z \in \mathbb{C}^{n},
$$

where $\wp: \mathbb{C}^{n} \rightarrow \mathbb{C}$ is an arbitrary holomorphic polynomial and $\beta \geq 0$ is a real number. According to Lemma 2.2 $u$ lies in $P S H_{n-1}(U)$, whenever the maxima $\theta_{1} \geq \theta_{2}$ below are equal for $\beta=0$ and any non-trivial compact ball $\bar{B} \subset U$ and arbitrary holomorphic polynomial $\wp$,

$$
\theta_{1}:=\max _{\bar{B}}\left(u-\varphi_{0}\right) \quad \text { and } \quad \theta_{2}:=\max _{b B}\left(u-\varphi_{0}\right) .
$$

Notice that both $\theta_{1}$ and $\theta_{2}$ exist, because $u$ is upper semi-continuous, but $\theta_{1}$ or $\theta_{2}$ may be equal to $-\infty$. Suppose by way of contradiction that $\theta_{1}>\theta_{2}$, so that the restriction of $u-\varphi_{0}$ to $\bar{B}$ does not attain its maximum $\theta_{1} \in \mathbb{R}$ at the boundary $b B$. One can easily choose $\beta>0$ small enough, so that the restriction of $u-\varphi_{\beta}$ to $\bar{B}$ does not attain its maximum at $b B$ either, but this maximum $\theta_{3} \in \mathbb{R}$ is 
indeed attained at some point $\hat{p}$ in the interior $B$. Lemma 2.8 yields the existence of $\psi \in \mathcal{C}^{2}(U)$ such that $u-\psi$ also has its strict global maximum at $\hat{p} \in U$ and the complex Hessians $H^{\mathbb{C}} \psi(\hat{p})$ and $H^{\mathbb{C}} \varphi_{\beta}(\hat{p})$ coincide. Since $u$ is $(n-1)$-plurisubharmonic in the viscosity sense on $U$, the Hessians $H^{\mathbb{C}} \psi=H^{\mathbb{C}} \varphi_{\beta}$ must have at most $n-1$ strictly negative eigenvalues at the point $\hat{p}$ according to Definition 2.6. We obviously have a contradiction, because $H^{\mathbb{C}} \varphi_{\beta}$ in (2.3) is equal to $-\beta<0$ times the $[n \times n]$-identity matrix. Whence, $\theta_{1}=\theta_{2}$ in (2.4), and so $u$ lies in $P S H_{n-1}(U)$ according to Lemma 2.2

We now consider the case when $0 \leq q \leq n-2$. Let $\pi \subset \mathbb{C}^{n}$ be any $(q+1)$ dimensional complex affine space such that $U \cap \pi$ is not empty. We can assume without loss of generality that $\pi$ is of the form $\mathbb{C}^{q+1} \times\{0\}$ for some particular system of coordinates $(x, y)$ in $\mathbb{C}^{q+1} \times \mathbb{C}^{n-q-1}$, since the eigenvalues of complex Hessians are invariant under holomorphic rotations and translations. Obviously, we have nothing to prove when $u$ is identically equal to $-\infty$ in $U \cap \pi$; otherwise, we follow the ideas presented in the previous paragraphs. Thus, $u \in P S H_{q}(U)$, whenever the maxima $\theta_{1} \geq \theta_{2}$ below are equal for any holomorphic polynomial $\wp: \mathbb{C}^{n} \rightarrow \mathbb{C}$ and non-empty open ball $B \subset \mathbb{C}^{q+1}$ with product $\bar{B} \times\{0\}$ contained in $U \cap \pi$,

$$
\theta_{1}:=\max _{\bar{B} \times\{0\}} \Upsilon \text { and } \theta_{2}:=\max _{b B \times\{0\}} \Upsilon \text { for } \Upsilon:=u-\operatorname{Re}[\wp] .
$$

Notice that both $\theta_{1}$ and $\theta_{2}$ exist, because $u$ is upper semi-continuous, but $\theta_{1}$ or $\theta_{2}$ may be equal to $-\infty$. Suppose by way of contradiction that $\theta_{1}>\theta_{2}$, so that the restriction of $\Upsilon$ to $\bar{B} \times\{0\}$ does not attain its maximum $\theta_{1} \in \mathbb{R}$ at the subset $b B \times\{0\}$. Since $\Upsilon$ is also upper semi-continuous, we can find a second non-empty open ball $D$ in $\mathbb{C}^{n-q-1}$ with centre at the origin and radius small enough, such that

$$
\overline{B \times D} \subset U \text { and } \max _{\overline{B \times D}} \Upsilon>\theta_{1}>\max _{b B \times \bar{D}} \Upsilon>\theta_{2},
$$

where $\Upsilon=u-\operatorname{Re}[\wp]$. Now define for $z=(x, y) \in \mathbb{C}^{q+1} \times \mathbb{C}^{n-q-1}$,

$$
\varphi(x, y):=\operatorname{Re}[\wp](x, y)-\beta\|x\|^{2}+\delta\|y\|^{2} .
$$

Again, since $u$ is upper semi-continuous, we can choose $\beta>0$ small enough and $\delta>0$ large enough, so that the restriction of $u-\varphi$ to $\overline{B \times D}$ does not attain its maximum at the boundary $b(B \times D)$ either, but this maximum $\theta_{3} \in \mathbb{R}$ is indeed attained at some point $\hat{p}$ in the interior $B \times D$. Lemma 2.8 yields the existence of $\psi \in \mathcal{C}^{2}(U)$ such that $u-\psi$ also has its strict global maximum at $\hat{p} \in U$ and the complex Hessians $H^{\mathbb{C}} \psi(\hat{p})$ and $H^{\mathbb{C}} \varphi(\hat{p})$ coincide. Since $u$ is $q$-plurisubharmonic in the viscosity sense on $U$, the Hessians $H^{\mathbb{C}} \psi=H^{\mathbb{C}} \varphi$ must have at most $q$ strictly negative eigenvalues at the point $\hat{p}$ according to Definition 2.6. We obviously have a contradiction, because $H^{\mathbb{C}} \varphi(\hat{p})$ in (2.6) has $q+1$ strictly negative eigenvalues coming from the term $-\beta\|x\|^{2}$. Hence, $\theta_{1}=\theta_{2}$ in (2.5), and so $u$ lies in $P S H_{q}(U)$ according to Definition 2.1.

\section{BASIC PROPERTIES OF THE SUP-CONVOLUTION}

We have so far characterised the $q$-plurisubharmonic functions as upper semicontinuous viscosity subsolutions to the elliptic degenerate operator $\ominus$ introduced in Lemma 2.4. One of the main consequences of this result is that we can apply the theory of viscous solutions into the field of several complex variables, as it was initially done by Słodkowski and Tomassini in [17, but we must be careful, because the operator $\ominus$ is not continuous. We recommend again to the interested reader 
the manuscripts of Crandall [4, Crandall et al. [5, Koike [10, and Katzourakis 9], for a general introduction to the theory of viscous solutions.

One of the most important tools in the theory of viscosity solutions is the supconvolution introduced by Moreau in 1963 [11, 12. It plays the role represented by the classical integral convolution in the theory of linear differential equations. Actually, it is well known that the sup-convolution takes an upper semi-continuous function $u$ (which may not be integrable) and in general produces a continuous one $u^{\Phi}$. In particular, if $\Phi$ is of the form $-k\|\cdot\|^{2}$, the sup-convolution then satisfies $u^{\Phi} \geq u$, the sum $u^{\Phi}+k\|\cdot\|^{2}$ is convex, and the sequence $\left\{u^{\Phi}\right\}_{k=1}^{\infty}$ is non-increasing and converges pointwise to $u$ as $k \rightarrow \infty$; see for example Chapter 4 of 9 or Section 11 of 4. Moreover, one also has the so called magic property: the supconvolution of a viscosity subsolution is again a viscosity subsolution.

We include in this chapter a fast introduction to the sup-convolution and its properties for the sake of completeness, but the reader who already knows the results can skip this chapter. Recall the original Moreau definition [11, 12.

Definition 3.1. Let $u: X \rightarrow[-\infty, \infty)$ and $\Phi: \mathbb{C}^{n} \rightarrow[-\infty, \infty)$ be a pair of upper semi-continuous functions, where $X \subset \mathbb{C}^{n}$ is a non-empty set. The sup-convolution is defined below for every $y \in \mathbb{C}^{n}$,

$$
u_{X}^{\Phi}(y):=\sup _{x \in X}\{u(x)+\Phi(y-x)\} \in[-\infty, \infty] .
$$

We simply write $u^{\Phi}$ instead of $u_{X}^{\Phi}$ when it is clear on which set the function $u$ is defined.

Even when the sup-convolution is well defined for every $y \in \mathbb{C}^{n}$, the most interesting results appear at those points $y \in \mathbb{C}^{n}$ for which the supremum in (3.1) can be attained at some $\hat{x} \in X$. Thus, we recall the semiconvex functions and the proper sets of definition for the sup-convolution.

Definition 3.2. Let $u: X \rightarrow[-\infty, \infty)$ and $\Phi: \mathbb{C}^{n} \rightarrow[-\infty, \infty)$ be both upper semi-continuous, where $X \subset \mathbb{C}^{n}$ is non-empty. The proper set of definition for the sup-convolution $u_{X}^{\Phi}$ is composed by those points $y \in \mathbb{C}^{n}$ for which there is $\hat{x} \in X$, such that the supremum $u_{X}^{\Phi}(y)$ in (3.1) is equal to $\Phi(y-\hat{x})+u(\hat{x})$.

Besides, a function $\Psi: \mathbb{C}^{n} \rightarrow \mathbb{R} \cup\{\infty\}$ is said to be semiconvex with constant $\delta>0$ if and only if the sum $\Psi+\delta\|\cdot\|^{2}$ is convex on $\mathbb{C}^{n}$.

Notice that the classical definition for convexity is extended to consider functions with image in $\mathbb{R} \cup\{\infty\}$. The sup-convolution has some simple but interesting properties. The proofs are included for the sake of completeness.

Lemma 3.3. Let $u: X \rightarrow[-\infty, \infty)$ and $\Phi: \mathbb{C}^{n} \rightarrow[-\infty, \infty)$ be a pair of upper semi-continuous functions, where $X \subset \mathbb{C}^{n}$ is a non-empty set. The statements below hold up for the sup-convolution $u^{\Phi}=u_{X}^{\Phi}$ in (3.1).

(1) The inequality $u \leq u^{\Phi}$ is satisfied on $X$, whenever $\Phi(0)=0$.

(2) If $\Phi \leq 0$ on $\mathbb{C}^{n}$ and there is $M \in \mathbb{R}$ with $u \leq M$ on $X$, then $u^{\Phi} \leq M$ is also bounded from above on $\mathbb{C}^{n}$ by the same constant.

(3) One has that $u_{X}^{\Phi}>-\infty$ on $\mathbb{C}^{n}$ if and only if neither $\Phi$ nor $u$ is identically equal to $-\infty$ on their respective set of definition.

(4) Let $Y \subset \mathbb{C}^{n}$ be the proper set of definition for $u_{X}^{\Phi}$. If neither $\Phi$ nor $u$ is identically equal to $-\infty$, then the image $u_{X}^{\Phi}(Y) \subset \mathbb{R}$ does not contain the extremal values $\pm \infty$. 
(5) If $\Phi \in \mathcal{C}\left(\mathbb{C}^{n}\right)$ is semiconvex with constant $\delta>0$ on $\mathbb{C}^{n}$ and $u \not \equiv-\infty$, then $u_{X}^{\Phi}$ is also semiconvex with constant $\delta>0$ on $\mathbb{C}^{n}$.

(6) Let $f: \mathbb{R} \rightarrow[-\infty, \infty)$ be upper semi-continuous and monotonically decreasing. Assume that $X \subset \mathbb{C}^{n}$ is closed, dist $_{X}$ is the Euclidean distance to $X$, and $\Phi=f(\|\cdot\|)$. The following identities hold on $y \in \mathbb{C}^{n}$,

$$
\chi_{\mathbb{C}^{n}}^{\Phi}=\chi_{X}^{\Phi}=f \circ \operatorname{dist}_{X} \quad \text { for } \quad \chi(y):=\left\{\begin{array}{cl}
0 & \text { if } y \in X \\
-\infty & \text { otherwise. }
\end{array}\right.
$$

Recall that $\langle x, y\rangle=\sum_{k=1}^{n} \operatorname{Re}\left(x_{k} \overline{y_{k}}\right)$ denotes the standard real inner product between vectors $x$ and $y$ in $\mathbb{C}^{n}$. Moreover, the notation $u \equiv-\infty$ means that $u$ is identically equal to $-\infty$ on its set of definition $X$.

Proof. The inequality $u \leq u^{\Phi}$ in statement (1) holds up after fixing $x=y$ into formula (3.1). The inequality $u^{\Phi} \leq M$ in point (2) is proved by setting $u \leq M$ into (3.1), so that for every $y \in \mathbb{C}^{n}$

$$
u^{\Phi}(y) \leq \sup _{x \in X}\{M+\Phi(y-x)\} \leq M .
$$

The result in point (3) follows from the fact that the sup-convolution $u^{\Phi}$ is calculated with the supremum. The result in statement (4) is proved by cases. Given any point $y$ in the proper set of definition for $u_{X}^{\Phi}$, there is $\hat{x} \in X$ such that $u_{X}^{\Phi}(y)$ is equal to $u(\hat{x})+\Phi(y-\hat{x})$. Thus, $u_{X}^{\Phi}(y)<\infty$, because $u<\infty$ and $\Phi<\infty$ on their respective sets of definition. Besides, point (3) yields that $u_{X}^{\Phi}>-\infty$ on $\mathbb{C}^{n}$, because neither $\Phi$ nor $u$ is identically equal to $-\infty$.

Definition 3.2 and the semiconvexity in the hypotheses of statement (5) easily yield that $\Phi \not \equiv-\infty$ and the sum $\Phi+\delta\|\cdot\|^{2}$ is convex on $\mathbb{C}^{n}$ for some $\delta>0$. Hence, we also have that $u_{X}^{\Phi}(y)+\delta\|y\|^{2}$ is convex with respect to the variable $y \in \mathbb{C}^{n}$, because it is the supremum of the following convex functions,

$$
\begin{aligned}
y \mapsto & u(x)-\delta\|x\|^{2}+2 \delta\langle x, y\rangle+\Phi(y-x)+\delta\|y-x\|^{2} \\
& =u(x)+\Phi(y-x)+\delta\|y\|^{2},
\end{aligned}
$$

which are all indexed by those points $x \in X$ with $u(x) \in \mathbb{R}$. Finally, point (6) easily follows from the definition of the distance function and the hypothesis that $f$ is monotonically decreasing; i.e.,

$$
\chi_{\mathbb{C}^{n}}^{\Phi}(y)=\sup _{x \in \mathbb{C}^{n}}\{\chi(x)+f(\|y-x\|)\}=\sup _{x \in X} f(\|y-x\|)=f\left(\operatorname{dist}_{X}(y)\right) .
$$

As we have said, one of the properties of the sup-convolution is that it takes upper semi-continuous functions (which may not be integrable) and produces continuous functions. This property is obtained when $\Phi$ is semiconvex; see Definition 3.2 .

Lemma 3.4. Let $X \subset \mathbb{C}^{n}$ be non-empty, and $u: X \rightarrow[-\infty, \infty)$ be upper semicontinuous. Given a semiconvex function $\Phi \in \mathcal{C}\left(\mathbb{C}^{n}\right)$ with constant $\delta>0$, so that $\Phi \not \equiv-\infty$, assume the proper set of definition for the sup-convolution $u_{X}^{\Phi}$ has nonempty interior $W \neq \emptyset$. The restriction of $f=u_{X}^{\Phi}+\delta\|\cdot\|^{2}$ to $W$ is then locally convex and continuous. Besides, if $u \not \equiv-\infty$, the function $f$ is twice differentiable almost everywhere on $W$ in the sense of Alexandrov; i.e., for almost every $x \in W$ there are a vector $\nu \in \mathbb{C}^{n}$ and a symmetric matrix $\mathcal{A} \in \mathbb{R}^{2 n \times 2 n}$ such that

$$
\lim _{h \rightarrow 0} \frac{f(x+h)-f(x)-\langle\nu, h\rangle-\left(h^{t} \mathcal{A} h\right) / 2}{\|h\|^{2}}=0 .
$$


In particular, if $f$ is $\mathcal{C}^{2}$-differentiable on a small neighbourhood of $x$ in $W$, one automatically has that $\nu=\nabla f(x)$ is the gradient and $\mathcal{A}=H^{\mathbb{R}} f(x)$ is the real Hessian; moreover $\mathcal{A} \geq 0$, because $f$ is locally convex at $x$.

Proof. The result is trivial when $u \equiv-\infty$, because $u_{X}^{\Phi} \equiv-\infty$ would be constant. Thus, we suppose from now on that it is not the case. Definition 3.1 and the point (5) in Lemma 3.3 imply that $f=u_{X}^{\Phi}+\delta\|\cdot\|^{2}$ is convex on $\mathbb{C}^{n}$, but it may take the extremal values $\pm \infty$. The point (4) in the same Lemma 3.3 then yields that $f(W) \subset \mathbb{R}$ does not contain the extremal values $\pm \infty$, so that the restriction of $f$ to $W$ is locally convex and continuous. The latter results are a consequence of Alexandrov's theorem for convex functions; see [1, 5].

Even when the sup-convolution $u^{\Phi}$ can be defined for all upper semi-continuous functions $u$ and $\Phi$, the more interesting cases happen when the proper set of definition for $u^{\Phi}$ is not empty and $u^{\Phi}$ is itself a continuous function. These properties are partially satisfied when $\Phi(x)$ is of the form $-\theta\|x\|^{2}$ for some positive real $\theta>0$ and according to Definition 3.2 Lemma 3.4, and the result below.

Lemma 3.5. Let $u: U \rightarrow[-\infty, M]$ be an upper semi-continuous function bounded from above by $M \in \mathbb{R}$ on a non-empty open set $U \subset \mathbb{C}^{n}$. Define $F_{\theta}=u_{U}^{\Phi}$ for the quadratic function $\Phi=-\theta\|\cdot\|^{2}$ and the real parameter $\theta>0$. Given any point $y \in U$, it lies in the proper set of definition for the sup-convolution $F_{\theta}$, whenever the image $u(y) \in \mathbb{R}$ is finite and the parameter $\theta \gg 1$ is large enough. Moreover, the following identity holds for every $y \in U$,

$$
u(y)=\lim _{\theta \rightarrow \infty} F_{\theta}(y)
$$

Proof. The result is trivial when $u \equiv-\infty$, because $F_{\theta}=u_{U}^{\Phi}$ would be constant and identically equal to $-\infty$. Thus, we suppose from now on that it is not the case. A direct consequence is deduced from statements (2) and (3) in Lemma 3.3. the image of $F_{\theta}=u_{U}^{\Phi}$ lies inside the real interval $(-\infty, M]$. Moreover, given any fixed point $y \in U$, the supremum in the Definition 3.1 of the sup-convolution $F_{\theta}(y)$ only needs to be calculated in the intersection of $U$ with a compact ball $\overline{B_{\rho}}(y)$ of centre at $y$ and radius $\rho>0$ large enough; i.e.,

$$
F_{\theta}(y)=\sup \left\{u(x)-\theta\|y-x\|^{2}: x \in U \cap \overline{B_{\rho}}(y)\right\}
$$

for any $\rho^{2}>\frac{M-F_{\theta}(y)}{\theta} \geq 0$, because $u(x)-\theta\|y-x\|^{2}<F_{\theta}(y)$ whenever $x \in U$ lies in the complement of $\overline{B_{\rho}}(y)$. Assume for some moments that $u(y) \in \mathbb{R}$ is finite for $y \in U$ fixed, the statement (11) in Lemma 3.3 implies that the sup-convolution $F_{\theta}(y)$ is bounded from below by $u(y)$ for every $\theta>0$. Whence, if we take the parameter $\theta \gg 1$ large enough in (3.5), we can chose a radius $\rho>0$ small enough such that the compact ball $\overline{B_{\rho}}(y)$ is contained in $U$. Since $u$ is upper semi-continuous, the maximum in (3.1) and 3.5 is attained at some point in $\overline{B_{\rho}}(y)$, and so $y$ lies in the proper set of definition for $F_{\theta}=u_{U}^{\Phi}$ according to Definition 3.2

Finally, we show (3.4) by considering two cases. If $u(y) \in \mathbb{R}$ is finite for $y \in U$ fixed, we have already seen that $F_{\theta}(y)$ is bounded from below by $u(y)$ for every $\theta>0$ according to point (11) in Lemma 3.3 . Hence, $\frac{M-F_{\theta}(y)}{\theta}$ converges to zero when $\theta$ goes to $\infty$, and so we can apply the limit when $\rho \rightarrow 0$ into (3.5) in order to deduce (3.4). Since $u$ is upper semi-continuous, we easily have that

$$
u(y) \leq \lim _{\theta \rightarrow \infty} F_{\theta}(y) \leq \lim _{\rho \rightarrow 0} \sup \left\{u(x): x \in U \cap \overline{B_{\rho}}(y)\right\} \leq u(y) .
$$


We proceed by contradiction when $u(y)=-\infty$ for the point $y \in U$. Assume that $F_{\theta}(y)$ in (3.4) does not converge to $-\infty$ when $\theta \rightarrow \infty$. It is easy to deduce from (3.1) or 3.5) that the sup-convolution $F_{\theta}(y)$ is monotonically decreasing with respect to $\theta$, and so $F_{\theta}(y)$ is bounded from below by some $\beta \in \mathbb{R}$ for every $\theta>0$. We may now proceed as in the previous paragraphs to deduce that equation (3.6) also holds. This result yields a contradiction, because $u(y)$ is equal to $-\infty$, and so both terms of equation (3.4) are equal to $-\infty$ as well.

\section{Sup-COnVOlution And $q$-PLURIsubharmonic FUnCtions}

As we mentioned in the introduction and the previous section, one of the most important results in the theory of viscosity solutions is the so called magic property: the sup-convolution $u^{\Phi}$ of a viscosity subsolution $u$ is again a viscosity subsolution (on the interior of the proper set of definition for $u^{\Phi}$ ). Thus, we conclude this work by showing below an extension to this magic property. As a pair of applications we also deduce that the sup-convolution of a strictly $q$-plurisubharmonic function is strictly $q$-plurisubharmonic and we find a new characterisation for the open $q$ pseudoconvex subsets $U \subset \mathbb{C}^{n}$. This latter result extends a list of characterisations and properties of $q$-pseudoconvex sets we collected in [13] and [14].

Proposition 4.1. Let $\Phi: \mathbb{C}^{n} \rightarrow[-\infty, \infty)$ be upper semi-continuous. Given a nonempty open set $U \subset \mathbb{C}^{n}$ and a fixed Hermitian matrix $\mathcal{A} \in \mathbb{C}^{n \times n}$, take any function $g \in \mathcal{C}^{2}(U)$ whose complex Hessian $H^{\mathbb{C}} g \geq \mathcal{A}$ is uniformly bounded from below on $U$; i.e., the difference $H^{\mathbb{C}} g-\mathcal{A}$ is positive semidefinite on $U$. Moreover, given any function $u \in P S H_{q}(U)$ for a fixed integer $q \geq 0$, suppose the proper set of definition for the sup-convolution $[u+g]_{U}^{\Phi}$ has non-empty interior $W \neq \emptyset$. If the restriction of $[u+g]_{U}^{\Phi}$ to $W$ is upper semi-continuous, then $[u+g]_{U}^{\Phi}-h$ lies in $P S H_{q}(W)$ for every $h \in \mathcal{C}^{2}(W)$ with complex Hessian $H^{\mathbb{C}} h \leq \mathcal{A}$ uniformly bounded from above on $W$.

Recall the Löwner partial order " $\geq$ " between Hermitian matrices introduced in Lemma 2.4.

Proof. The result is trivial when $q \geq n$ or $[u+g]_{U}^{\Phi} \equiv-\infty$ on $W$. Thus, we assume from now on that $q<n$ and neither $\Phi$ nor $u$ is identically equal to $-\infty$ on their respective set of definition. Recall that $[u+g]_{U}^{\Phi} \equiv-\infty$ whenever $\Phi$ or $u$ is identically equal to $-\infty$ because of Definition 3.1 or the point (3) in Lemma 3.3. On the other hand, since $W$ is the interior of the proper set of definition for the supconvolution $[u+g]_{U}^{\Phi}$, its image $[u+g]_{U}^{\Phi}(W) \subset \mathbb{R}$ does not contain the extremal values $\pm \infty$ according to the point (4) in the same Lemma 3.3 .

Define now $F:=[u+g]_{U}^{\Phi}-h$ and consider any $\varphi \in \mathcal{C}^{2}(W)$ such that $F-\varphi$ takes its maximum at some $\hat{p} \in W$. We shall prove that $\ominus\left(H^{\mathbb{C}} \varphi\right) \leq q$ at the point $\hat{p}$, so that Definition 2.6 and Theorem 2.10 will imply that $F$ is $q$-plurisubharmonic in the classical and viscosity senses on $W$. Since $F-\varphi$ takes its maximum at $\hat{p} \in W$, formula (3.1) yields the identities below for all $w \in W$ and $y \in U$,

$$
\begin{aligned}
{[u+g]_{U}^{\Phi}(\hat{p})-} & (h+\varphi)(\hat{p}) \geq[u+g]_{U}^{\Phi}(w)-(h+\varphi)(w) \\
& \geq(u+g)(y)+\Phi(w-y)-(h+\varphi)(w) .
\end{aligned}
$$

Since $\hat{p}$ lies in the interior $W$ of the proper set of definition for $[u+g]_{U}^{\Phi}$, Definition 3.2 implies the existence of a point $\hat{x} \in U$, such that

$$
(u+g)(\hat{x})+\Phi(\hat{p}-\hat{x})=[u+g]_{U}^{\Phi}(\hat{p}) \in \mathbb{R} .
$$


Now fix $w=f(y):=y-\hat{x}+\hat{p}$, so that $w-y$ is equal to $\hat{p}-\hat{x}$. There is then a small enough neighbourhood $\Omega$ of $\hat{x}$ in $U$, such that $f(\Omega) \subset W$ is also a neighbourhood of $\hat{p}=f(\hat{x})$ in $W$. Equations (4.1) and (4.2) yield the following inequality for every point $y \in \Omega$,

$$
(u+g)(\hat{x})-(h+\varphi) \circ f(\hat{x}) \geq(u+g)(y)-(h+\varphi) \circ f(y) .
$$

Whence, if we define $\Upsilon:=-g+(h+\varphi) \circ f$, the restriction of $u-\Upsilon$ to $\Omega$ attains its maximum at $\hat{x} \in \Omega$. Since $u(\hat{x}) \in \mathbb{R}$ is finite according to (4.2), Lemma 2.8 implies the existence of a test function $\psi \in \mathcal{C}^{2}(U)$ such that $u-\psi$ has a strict global maximum at $\hat{x} \in U$ and the complex Hessians $H^{\mathbb{C}} \Upsilon(\hat{x})$ and $H^{\mathbb{C}} \psi(\hat{x})$ coincide. The given hypotheses also imply that $H^{\mathbb{C}} h(\hat{p}) \leq H^{\mathbb{C}} g(\hat{x})$, because $H^{\mathbb{C}} h \leq \mathcal{A}$ on $W$ and $\mathcal{A} \leq H^{\mathbb{C}} g$ on $U$; see for example section 6.2 of [19]. Since $f$ is a translation, we can use all the previous results to deduce that,

$$
H^{\mathbb{C}} \psi(\hat{x})=H^{\mathbb{C}} \Upsilon(\hat{x})=H^{\mathbb{C}}(h+\varphi)(\hat{p})-H^{\mathbb{C}} g(\hat{x}) \leq H^{\mathbb{C}} \varphi(\hat{p}) .
$$

Now then, the operator $\ominus\left(H^{\mathbb{C}} \psi\right) \leq q$ at the point $\hat{x}$, because $u-\psi$ has a strict global maximum at $\hat{x} \in U$ and $u \in P S H_{q}(U)$. Recall the given hypotheses, Definition 2.6. and Theorem 2.10. Moreover, $\ominus\left(H^{\mathbb{C}} \varphi\right) \leq q$ at $\hat{p} \in W$ because of (4.3) and Lemma 2.4, so that the restriction of $[u+g]_{U}^{\Phi}-h$ to $W$ is $q$-plurisubharmonic in the classical and viscosity senses on $W$.

We can obviously define $g(y)=h(y)=y^{t} \mathcal{A} \bar{y}$ in the previous proposition for any fixed Hermitian matrix $A \in \mathbb{C}^{n \times n}$, so that the complex Hessians $H^{\mathbb{C}} g$ and $H^{\mathbb{C}} h$ given in 2.1) are constant and equal to $\mathcal{A}$. The following results are a direct consequence of Lemma 3.4 and Proposition 4.1.

Corollary 4.2. Let $\Phi \in \mathcal{C}\left(\mathbb{C}^{n}\right)$ be semiconvex with constant $\delta>0$, and $u$ be $q$-plurisubharmonic on a non-empty open set $U \subset \mathbb{C}^{n}$ and for an integer $q \geq 0$. Given a constant Hermitian matrix $\mathcal{A} \in \mathbb{C}^{n \times n}$, define $g(y)=y^{t} \mathcal{A} \bar{y}$ and suppose that the proper set of definition for the sup-convolution $[u+g]_{U}^{\Phi}$ has non-empty interior $W \neq \emptyset$. The restriction of $[u+g]_{U}^{\Phi}-g$ to $W$ is then continuous and q-plurisubharmonic on $W$.

Observe that $\Phi \not \equiv-\infty$ because of the given hypotheses. Moreover, one can fix $g \equiv 0$ in Corollary 4.2, so that the sup-convolution $u^{\Phi}$ of a $q$-plurisubharmonic function $u$ is again $q$-plurisubharmonic. We can show a similar result for strictly $q$-plurisubharmonic functions. Recall the following definition from [3, 18, 6].

Definition 4.3. Let $u: U \rightarrow[-\infty, \infty)$ be upper semi-continuous on a non-empty open set $U \subset \mathbb{C}^{n}$. One says that $u$ is strictly $q$-plurisubharmonic on $U$ if and only if for every point $y \in U$ there are a real $\varepsilon>0$ and a non-empty open ball $B \subset U$ with centre at $y$, such that the restriction of $u-\varepsilon\|\cdot\|^{2}$ to $B$ is $q$-plurisubharmonic on $B$.

Notice that $u \equiv-\infty$ is strictly $q$-plurisubharmonic; and it is the only constant function with this property. One must also say that the strictly q-plurisubharmonic functions are sometimes called strongly q-plurisubharmonic.

Corollary 4.4. Let $u: U \rightarrow[-\infty, \infty)$ be strictly q-plurisubharmonic on a nonempty open set $U \subset \mathbb{C}^{n}$ and for an integer $q \geq 0$. Given any compactly contained open set $\Omega \Subset U$, assume the proper set of definition for the sup-convolution $\left[\left.u\right|_{\Omega}\right]_{\Omega}^{\Phi}$ has a non-empty interior $W \neq \emptyset$, where $\Phi \in \mathcal{C}\left(\mathbb{C}^{n}\right)$ is semiconvex with constant 
$\delta>0$. The restriction of $\left[\left.u\right|_{\Omega}\right]_{\Omega}^{\Phi}$ to $W$ is then continuous and strictly q-plurisubharmonic on $W$.

Proof. We assert that the restriction of $u-\delta\|\cdot\|$ to $\Omega$ is $q$-plurisubharmonic on $\Omega$ for some small real $\delta>0$. Since $\bar{\Omega} \subset U$ is a compact set, Definition 4.3 yields the existence of $m$ positive real numbers $\left\{\varepsilon_{j}\right\}_{j=1}^{m}$ and a finite cover $\left\{B_{j}\right\}_{j=1}^{m}$ of $\bar{\Omega}$ by non-empty open balls, such that the restriction of $u-\varepsilon_{j}\|\cdot\|^{2}$ to $B_{j}$ is $q$-plurisubharmonic on $B_{j}$ for every $j=1, \ldots, m$. The real $\delta>0$ we are looking for is the minimum of $\left\{\varepsilon_{j}\right\}_{j=1}^{m}$.

Corollary 4.2 now implies that the restriction of $\left[\left.u\right|_{\Omega}\right]_{\Omega}^{\Phi}-\varepsilon\|\cdot\|$ to $W$ is also continuous and $q$-plurisubharmonic on $W$, because we only need to fix $g=\varepsilon\|\cdot\|$. Hence, $\left[\left.u\right|_{\Omega}\right]_{\Omega}^{\Phi}$ is strictly $q$-plurisubharmonic on $W$.

Another interesting result is that the complex Hessian $H^{\mathbb{C}} u$ of a strictly $q$-plurisubharmonic function $u$ has at least $n-q$ strictly positive eigenvalues in the viscous sense. Recall the operator $\oplus$ defined in Lemma 2.4.

Lemma 4.5. Let $u: U \rightarrow[-\infty, \infty)$ be strictly q-plurisubharmonic on an open set $U \subset \mathbb{C}^{n}$ and for an integer $q \geq 0$. Assume that $u \not \equiv-\infty$. Given any test function $\varphi \in \mathcal{C}^{2}(U)$, the operator $\oplus\left(H^{\mathbb{C}} \varphi\right) \geq n-q$ at those points $\hat{p} \in U$ where $u-\varphi$ attains its maximum; i.e., the complex Hessian $H^{\mathbb{C}} \varphi$ in (2.1) has at least $n-q$ strictly positive eigenvalues at those points $\hat{p}$.

Proof. The result is trivial when $q \geq n$, so we suppose it is not the case. According to Definition 4.3 and Theorem 2.10 there are a real $\varepsilon>0$ and a non-empty open ball $B \subset U$ with centre at $y$, such that the restriction of $u-\varepsilon\|\cdot\|^{2}$ to $B$ is $q$-plurisubharmonic in the classical and viscosity senses on $B$. Since the restriction of $u-\varphi$ to $\Omega$ also takes its maximum at $\hat{p} \in \Omega$, we can add and subtract $\varepsilon\|\cdot\|^{2}$ to $u-\varphi$ and consider the new test function $\varphi-\varepsilon\|\cdot\|^{2}$, so that Definition 2.6 yields,

$$
q \geq \ominus\left(H^{\mathbb{C}}\left(\varphi-\varepsilon\|\cdot\|^{2}\right)(\hat{p})\right)=\ominus\left(H^{\mathbb{C}} \varphi(\hat{p})-\varepsilon \mathbb{1}\right),
$$

where $\mathbb{1}$ is the identity $[n \times n]$-matrix. Hence, $H^{\mathbb{C}} \varphi(\hat{p})-\varepsilon \mathbb{1}$ has at most $q$ strictly negative eigenvalues, and so $H^{\mathbb{C}} \varphi(\hat{p})$ has at least $n-q$ strictly positive eigenvalues, because $\varepsilon>0$.

Unfortunately, the converse of the previous lemma does not hold in general.

Example 4.6. The function $\operatorname{Im}^{4}(z)=\frac{(z-\bar{z})^{4}}{16}$ obviously lies in $P S H_{0}(\mathbb{C})$, but it is only strictly plurisubharmonic on $\mathbb{C} \backslash \mathbb{R}$, because its complex Hessian is $3 \operatorname{Im}^{2}(z)$ and it vanishes on the real axis $\mathbb{R} \subset \mathbb{C}$. In the same way, the point (3) in Proposition 2.3 implies that

$$
f(z):=\operatorname{Im}^{4}(z)+|\operatorname{Im}(z)|=\operatorname{Im}^{4}(z)+\max \{\operatorname{Im}(z),-\operatorname{Im}(z)\}
$$

lies in $P S H_{0}(\mathbb{C})$. We easily have $H^{\mathbb{C}} f(z)=3 \operatorname{Im}^{2}(z)$ on $\mathbb{C} \backslash \mathbb{R}$, so that $f$ is also strictly plurisubharmonic on $\mathbb{C} \backslash \mathbb{R}$. Nevertheless, $f$ is not strictly plurisubharmonic on $\mathbb{R}$, because for every $\varepsilon>0$ the complex Hessian of $f-\varepsilon\|\cdot\|^{2}$ converges to $-\varepsilon$ when $z \notin \mathbb{R}$ moves close enough to $\mathbb{R}$, so it becomes strictly negative near $\mathbb{R}$.

On the other hand, let $\varphi \in \mathcal{C}^{2}(\mathbb{C})$ be any test function, such that $f-\varphi$ takes its maximum at some $\hat{p} \in \mathbb{C}$. Notice that $f-\varphi$ cannot take its maximum in the real axis $\mathbb{R} \subset \mathbb{C}$ because of the absolute value $|\operatorname{Im}(z)|$ in (4.4), so that $\hat{p} \notin \mathbb{R}$. Since 
$f-\varphi$ is $\mathcal{C}^{2}$-smooth on $\mathbb{C} \backslash \mathbb{R}$, we can repeat word by word the analysis done in the second part of the proof of Lemma 2.5 to deduce that

$$
H^{\mathbb{C}} \varphi(\hat{p}) \geq H^{\mathbb{C}} f(\hat{p})=3 \operatorname{Im}^{2}(\hat{p})>0
$$

at every point $\hat{p}$ where $f-\varphi$ takes its maximum.

Another interesting example appears after considering the result presented in the point (6) of Lemma 3.3. The sup-convolution $\chi^{\Phi}$ is equal to the composition of the distance $\operatorname{dist}_{X}$ with a monotonically decreasing function $f$. Whence, if the characteristic function $\chi$ in (3.2) or (4.5) below is $q$-plurisubharmonic, Lemma 3.3 and Proposition 4.1] would imply that $\chi^{\Phi}$ and $f \circ$ dist $_{X}$ are both $q$-plurisubharmonic as well. In this case, Słodkowski characterised in [16] those closed sets $X \subset \mathbb{C}^{n}$ for which its characteristic function $\chi \in P S H_{q}\left(\mathbb{C}^{n}\right)$. Słodkowski actually produced several characterisations in [16], one of them says that $\chi \in P S H_{q}\left(\mathbb{C}^{n}\right)$ if and only if the open complement $\mathbb{C}^{n} \backslash X$ is $(q-1)$-pseudoconvex; see for example Theorems 2.5 and 4.2 in [16] or Theorem 3.8 in [14. We can restate the previous analysis as follows.

Corollary 4.7. Let $f: \mathbb{R} \rightarrow[-\infty, \infty)$ be upper semi-continuous and monotonically decreasing, such that $f(0)=0$ and $f(t)<0$ for all $t>0$. Take the Euclidean distance dist $_{X}$ to a non-empty closed set $X \subset \mathbb{C}^{n}$. Given any integer $q \geq 0$, the statements below are all equivalent:

(1) The characteristic function below lies in $\mathrm{PSH}_{q}\left(\mathbb{C}^{n}\right)$,

$$
\chi(y):=\left\{\begin{array}{cl}
0 & \text { if } y \in X ; \\
-\infty & \text { otherwise. }
\end{array} \quad \text { for } \quad y \in \mathbb{C}^{n} .\right.
$$

(2) The composition $f \circ$ dist $_{X}$ lies in $P S H_{q}\left(\mathbb{C}^{n}\right)$.

(3) The open set $\mathbb{C}^{n} \backslash X$ is (q-1)-pseudoconvex on $\mathbb{C}^{n}$ under the extra condition that $1 \leq q<n$, and so $-\ln \operatorname{dist}_{X}$ lies in $P S H_{q-1}\left(\mathbb{C}^{n} \backslash X\right)$.

Notice that $\chi$ in (4.5) is upper semi-continuous when $X \subset \mathbb{C}^{n}$ is closed. Moreover, if we set $q=1$ in the above statements, we obtain a new characterisation for domains of holomorphy (0-plurisubharmonic domains) $D \subset \mathbb{C}^{n}$ : The composition $f \circ$ dist $_{X}$ is 1-plurisubharmonic for the distance dist $x_{X}$ to the complement $\mathbb{C}^{n} \backslash D$ and a monotonically decreasing function $f$ as above.

Proof. (11) implies (2). We have $\chi^{\Phi}=f \circ \operatorname{dist}_{X}$ for $\Phi=f(\|\cdot\|)$ according to the point (6) in Lemma 3.3 and identity (3.3). Moreover, $\mathbb{C}^{n}$ is the proper set of definition for $\chi^{\Phi}$, because $X$ is closed, so that for each $y \in \mathbb{C}^{n}$ there is $\hat{x} \in X$ with $\operatorname{dist}_{X}(y)$ equal to $\|\hat{x}-y\|$. Since $f \circ \operatorname{dist}_{X}$ is upper semi-continuous and $\chi$ lies in $\mathrm{PSH}_{q}\left(\mathbb{C}^{n}\right)$ by hypothesis, Proposition 4.1 imply that $\chi^{\Phi}=f \circ \operatorname{dist}_{X}$ lies in $P S H_{q}$ as well.

(2) implies (11). Notice that $\chi=\lim _{k \rightarrow \infty} u_{k}$ for $u_{k}:=k\left(f \circ \operatorname{dist}_{X}\right)$ and every integer number $k \in \mathbb{N}$. Since $u_{1} \geq u_{2} \geq \ldots$, points (3) and (4) in Proposition 2.3 implies that $u_{k}$ and $\chi$ both lie in $\operatorname{PSH}_{q}\left(\mathbb{C}^{n}\right)$.

(11) is equivalent to (3). Theorems 2.5 and 4.2 in [16 state that $\chi$ lies in $\mathrm{PSH}_{q}\left(\mathbb{C}^{n}\right)$ if and only if the complement $\mathbb{C}^{n} \backslash X$ is $(q-1)$-pseudoconvex. Finally, the equivalence with the property that $-\ln \operatorname{dist}_{X}$ lies in $P S H_{q-1}\left(\mathbb{C}^{n} \backslash X\right)$ follows from Definition 3.3 in [13] or Proposition 3.3 in [14]. 
This result extends a list of characterisations and properties of $q$-pseudoconvex sets we collected in [13] and [14. Besiedes, we must point out that the previous results can be extended to consider locally closed sets $X \subset \mathbb{C}^{n}$ instead of closed ones. However, we must be careful, because the proper set of definition for $\chi^{\Phi}$ may not be the whole space $\mathbb{C}^{n}$. Indeed, for each $y \in \mathbb{C}^{n}$ there is $\hat{x} \in \bar{X}$ with $\operatorname{dist}_{X}(y)$ equal to $\|\hat{x}-y\|$, but $\hat{x}$ may not lie in $X$, when it is different from $\bar{X}$.

\section{REFERENCES}

[1] A. D. Alexandrov, Almost everywhere existence of the second differential of a convex function and some properties of convex surfaces connected with it, Leningrad State Univ. Annals [Uchenye Zapiski] Math. Ser., 6 (1939), pp. 3-35.

[2] O. Alvarez, J.-M. Lasry, And P.-L. Lions, Convex viscosity solutions and state constraints, J. Math. Pures Appl., 75 (1997), pp. 265-288.

[3] L. Bungart, Piecewise smooth approximations to q-plurisubharmonic functions, Pacific J. Math., 142 (1990), pp. 227-244.

[4] M. G. Crandall, Viscosity solutions: A primer, in Viscosity Solutions and Applications, vol. 1660 of Lecture Notes in Mathematics, Springer-Verlag Berlin, 1997, pp. 1-43.

[5] M. G. Crandall, H. Ishit, and P.-L. Lions, User's guide to viscosity solutions of second order partial differential equations, Bull. Amer. Math. Soc., 27 (1992), pp. 1-67.

[6] N. Q. Dieu, q-plurisubharmonicity and q-pseudoconvexity in $\mathbb{C}^{n}$, Publ. Mat., 50 (2006), pp. 349-369.

[7] O. Fujita, On the equivalence of the q-plurisubharmonic functions and the pseudoconvex functions of general order, Ann. Reports of Graduate School of Human Culture, Nara Women's Univ., 7 (1992), pp. 77-81.

[8] L. R. Hunt and J. J. Murray, q-plurisubharmonic functions and a generalized Dirichlet problem, Mich. Math. J., 25 (1978).

[9] N. Katzourakis, An Introduction To Viscosity Solutions for Fully Nonlinear PDE with Applications to Calculus of Variations in $L^{\infty}$, Springerbriefs in Mathematics, Springer, Berlin, 2015.

[10] S. Kolke, A Beginner's Guide to the Theory of Viscosity Solutions, vol. 13 of MSJ Memoirs, Mathematical Society of Japan, Saitama, 2013.

[11] J.-J. Moreau, Inf-convolution des fonctions numériques sur un espace vectoriel, C.R. Acad. Sci. Paris, 256 (1963), pp. 5047-5049.

[12] — Fonctionnelles convexes, Séminaire Jean Leray, 2 (1966-1967), pp. 1-108.

[13] T. PaWlaschyK and E. S. Zeron, On convex hulls and pseudoconvex domains generated by q-plurisubharmonic functions, part I, J. Math. Anal. App., 408 (2013), pp. 394-408.

[14] - On convex hulls and pseudoconvex domains generated by q-plurisubharmonic functions, part II, Bol. Soc. Mat. Mex., 22 (2016), pp. 367-388.

[15] Z. SŁodKowski, The Bremermann-Dirichlet problem for q-plurisubharmonic functions, Ann. Scuola Norm. Super. Pisa-Cl. Sci. (4), 11 (1984), pp. 303-326.

[16] - Local maximum property and q-plurisubharmonic functions in uniform algebras, J. Math. Anal. Appl., 115 (1986), pp. 105-130.

[17] Z. SŁodkowski And G. Tomassini, Weak solutions for the levi equation and envelope of holomorphy, J. Funct. Anal., 101 (1991), pp. 392-407.

[18] V. VÂJÂITU, Locally q-complete open sets in Stein spaces with isolated singularities, Kyushu J. Math., 51 (1997), pp. 355-368.

[19] F. Zhang, Matrix Theory: Basic Results and Techniques, Springer, Berlin, 1999.

University of Wuppertal, School of Mathematics and Natural Sciences, Gaussstrasse 20, 42119 Wuppertal, Germany

E-mail address: pawlaschyk@math.uni-wuppertal.de

Departamento de Matemáticas, Cinvestav del ipN, Apartado Postal 14-740, CiuDAD DE MÉXICO, 07000, MeXico

E-mail address: eszeron@math.cinvestav.edu.mx 